Method A total of 1348 subjects (1063 male and 285 female) who experienced polysomnography (PSG) during 2004-2010 in The Third Hospital of Peking University ENT department were included. The correlativity between apnoea-hypopnoea index (AHI) and BMI was conducted by pearson correlation test. The diagnostic value and reference standard in both male and female were analysed by ROC curve.

Result 1) A positive relationship between AHI and BMI was detected, both in male and female subjects, with pearson coefficients 0.423 and 0.419 ( $p<0.001$ ). 2) Results of ROC analysis show a favourable value of BMI in diagnosing OSHAS, especially in male subjects. In male population, area under curve (AUC) was 0.759 (95\% CI 0.724 to $0.795)$ with a best cutoff value $25.3 \mathrm{~kg} / \mathrm{m}^{2}$. The sensitivity and specificity in man were $74 \%$ and $61 \%$, respectively; in women, area under curve (AUC) was 0.691 (95\% CI 0.632 to 0.751 ) with a best cutoff value $24.7 \mathrm{~kg} / \mathrm{m}^{2}$. The sensitivity and specificity were $68 \%$ and $61 \%$. The screening cutoff values were $22.4 \mathrm{~kg} / \mathrm{m}^{2}$ and $20.7 \mathrm{~kg} / \mathrm{m}^{2}$ in male and female, with a sensitivity of $95 \%$. The diagnostic cutoff values were $30.0 \mathrm{~kg} / \mathrm{m}^{2}$ and $36.0 \mathrm{~kg} / \mathrm{m}^{2}$, with a specificity of $95 \%$.

Conclusion BMI seemed a better diagnostic value of OSAHS in diagnosing than screening, especially in male population. Sexualseparated cutoff values should be use in clinical practice to enhance diagnostic accuracy.

\section{e0313 THE DETECTION OF PLASMA VISFATIN IN OBESE PATIENTS WITH CORONARY ARTERY DISEASE}

doi:10.1136/hrt.2010.208967.313

Yu Qin, Hong-Jiu Yu, Tian Lv. Department of the First Hospital of Dalian Medical University

Objective To explore the level of plasma visfatin in patients with coronary artery disease (CAD) and different BMI, and evaluate the relationship between plasma visfatin and coronary lesion severity in obese patients with CAD

Methods 59 causes were undergone coronary angiography and divided into three sub-groups: normal weight (13 causes) $\left(\mathrm{BMI}<24 \mathrm{~kg} / \mathrm{m}^{2}\right)$, over weight (31 causes) $\left(24 \leq \mathrm{BMI}<28 \mathrm{~kg} / \mathrm{m}^{2}\right)$ and obese groups (15 causes) ( $\left.\mathrm{BMI} \geq 28 \mathrm{~kg} / \mathrm{m}^{2}\right)$. The levels of plasma visfatin of $\mathrm{CAD}$ group and control group were measured and compared. The correlation between plasma visfatin and coronary lesion severity score with different BMI was analysed.

Result The plasma visfatin level was higher in the CAD group $(547.21 \pm 94.18 \mathrm{ng} / \mathrm{ml})$ comparing with the control group $(470.86 \pm 87.99 \mathrm{ng} / \mathrm{ml})$. In obese patients with CAD the plasma visfatin level $(586.62 \pm 96.77 \mathrm{ng} / \mathrm{ml})$ increased more significantly and it was positively correlated with Gensini score $(p<0.05)$.

Conclusion The level of plasma visfatin is closely correlated with coronary lesion severity in obese patients with $\mathrm{CAD}$. It might be used as a new clinical marker to predict the severity of coronary lesion in obese patients with $\mathrm{CAD}$.

\section{e0314 ASSOCIATION OF OBESITY WITH RENIN-ANGIOTENSIN SYSTEM}

doi:10.1136/hrt.2010.208967.314

Ali Raza Kazmi, Muhammad Ismail, Mansoor Qaiser. Ibgeg-9/1 Islamabad

Overweight and obesity are highly associated with multiple comorbidities, elevated blood pressure values, dyslipidaemia, reduced insulin sensitivity and alterations of large and minor vessels. The essential role of the renin-angiotensin system (RAS) in controlling blood pressure has well been established. Genes encoding components of the RAS have been proposed as candidate genes that determine genetic predisposition to hypertension and the risk of developing cardiovascular complications. The role of these genes in obesity is not well understood. Several studies have showed their association with obesity in animal models.

\section{Clinical and Research Medicine: Cardiovas- cular Clinical Pharmaceutical Research e0315 EXPLORE THE RELATIONSHIP BETWEEN LEFT VENTRICULAR MURAL THROMBUS AND LEFT VENTRICULAR ANEURYSM AFTER ACUTE MYOCARDIAL INFARCTION}

doi:10.1136/hrt.2010.208967.315

Zhang Zhiping, Cheng Guohong, Su Xi. Department of Cardiology, Wuhan Asia Heart Hospital, Wuhan 430022, China

Objective To explore the relationship between left ventricular mural thrombus (LVMT) and left ventricular aneurysm (LVA) after acute myocardial infarction (AMI).

Methods The clinical materials of 66 consecutive patients with LVA after AMI were retrospectively analysed. These patients were divided into two groups according the result of LVMT formation diagnosed by echocardiographic. 30 cases with LVMT entered group LVMT and the other 36 cases entered group without LVMT. The mean left atrial (LA), LV end-diastolic and end-systolic diameter, thickness of the interventricular septum (IVS) and LV posterior wall (PW), and the size of the aneurysm as well as the LV ejection fraction (LVEF) were compared among two groups.

Results According to results of analysis, the mean LA, LV enddiastolic and end-systolic diameter, PW thickness, and the size of the aneurysm did not differ between patients with and without LVMT. The thickness of the IVS were greater in patients in with LVMT than in those without LVMT $(1.17 \pm 0.26$ vs $1.04 \pm 0.19 \mathrm{~cm}$, respectively; $\mathrm{p}=0.02$ ).

Conclusions Left ventricular aneurysm formation after AMI could be related to LVMT. However, the size of the aneurysm was not significant associated with LVMT.

\section{e0316 THE scD40L AND CIRCULATION ENDOTHELIAL PROGENITOR CELLS CHANGE OF DANHONG COMBINED NAOXINTONG THERAPY TO ACUTE CORONARY SYNDROME PATIENTS WITH PERCAUTIOUS CORONARY INTERVENTION}

doi:10.1136/hrt.2010.208967.316

${ }^{1}$ Lu Jing-qian, ${ }^{2}$ Li Yi, ' Lv Yun, ${ }^{1}$ Pan Ya-ping, ' ${ }^{1}$ Gao Yan, ${ }^{1}$ Yang Feng. ${ }^{1}$ Department of Cardiologythe 3th Hospital of Yunnan Province; ${ }^{2}$ Saints Heart Hospital of Yunnan

Objective To observe the effects of Danhong injection combined naoxintong pills with acute coronary syndrome (ACS) patients who accepted percautious coronary intervention (PCI) about recelently outcome and changes of blood serum soluble CD40 ligand (sCD40L) and Circulation Endothelial Progenitor Cells (EPCs).

Methods 91 ACS patients with PCI therapy were randomly divided into Buchang group $(n=48)$ and normaltherapy group $(n=43)$, after PCI, normaltherapy group were given coronary heart disease second prevention drugs, and Buchang group were added danghong and naoxintong at the basis of coronary heart disease second prevention drugs. the outcome after PCI 3 months, 6 months and changes of sCD40L and EPCs were observed.

Results In two groups, cardiac death, myocardial infarction, stroke, angina, re-hospitality were no significantly difference $(p>0.05)$ but the left ventricular ejection fraction were more higher in Buchang group than normaltherapy group $(61.5 \%$ vs $55.3 \%, \mathrm{p}<0.05)$. 6 miniters walk distence test were more higher in Buchang group 
than normaltherapy group (562 m vs $513 \mathrm{~m}, \mathrm{p}<0.05$ ) at 3 months after operate. Still after 6 months, the left ventricular ejection fraction were more higher in Buchang group than normaltherapy group $(65.3 \%$ vs $60.8 \%, p<0.05)$. 6 miniters walk distence test were more higher in Buchang group than normaltherapy group (541 m vs $502 \mathrm{~m}$, $p<0.05)$. Moreover after 3 months, the level of sCD40L were lower in Buchang group than normaltherapy group $(2.23 \mathrm{ng} / \mathrm{ml}$ vs $2.62 \mathrm{ng} / \mathrm{ml}$, $\mathrm{p}<0.01$ ), the numbers of EPCs were more in Buchang group than normaltherapy group $(37.1 \pm 5.8$ vs $32.4 \pm 4.6, \mathrm{p}<0.05)$.

Conclusion These study suggested that Danhong injection combined naoxintong pills could decreased SCD40L level, increased EPCs level, improved cardia function at ACS patients with PCI therapy, It was worthy for deeply research.

\section{e0317 COMPARATIVE EFFECTIVENESS OF RENIN ANGIOTENSIN SYSTEM BLOCKADES PLUS CCBS OR DIURETICS FOR ESSENTIAL HYPERTENSION A SYSTEMATIC REVIEW}

doi:10.1136/hrt.2010.208967.317

Ma Ruixin, Yu Jing, Xu Dian, Yang Longquan, Liu Peijun, Bai Feng. The Second Hospital of Lanzhou University

Backround The relative effectiveness of two combination therapyRenin Angiotensin System (RAS) blockades/calcium channel blockers (CCBs) versus RAS blockades/diuretics for lowering blood pressure is unknown. This systematic review is to compare the benefits and harms of RAS blockades plus CCBs versus RAS blockades plus diuretics for treating essential hypertension in adults. Methods We retrieved MEDLINE, the Cochrane Central Register of Controlled Trials, EMBASE and SCI using computer to identify relevant randomised controlled trials in English that directly compared the effect of RAS blockades plus CCBs with that of RAS blockades plus diuretics in adult patients with essential hypertension, reported an outcome of mean difference of BP reduction or interest, lasted at least 4 weeks, and included at least 20 patients. A standardised protocol with predefined criteria was used to extract data on study design, interventions, population characteristics, and outcomes; We evaluated study quality and applicability; and assessed the strength of the evidence for key outcomes.

Results Five clinical studies were eventually included. We found no significant difference between RAS blockades/CCBs with RAS blockades/diuretics in reduction of blood pressure. However, RAS blockade/ $\mathrm{CCBs}$ associated with significant stronger DBP response rate. No differential effects were observed for the incidence of adverse events. Conclusion Available evidence shows that RAS blockade/CCBs and blockade/diuretics have similar effects on blood pressure control. High strength of evidence is needed. Data regarding is patient subgroups were missing.

\section{e0318 PITAVASTATIN CALCIUM IMPROVES CAROTID ARTERIOSCLEROSIS IN PATIENTS WITH HYPERCHOLESTEROLAEMIA}

doi:10.1136/hrt.2010.208967.318

Yan Hui-Min, Wang Jia, Wang Hua, Wang Zhi-Hao, Zhang Wei, Zhang Yun, Zhong Ming. Key Laboratory of Cardiovascular Remodelling and Function Research, Chinese Ministry of Education and Chinese Ministry of Public Health, Department of Cardiology, Qilu Hospital of Shandong University, Jinan, China

Objective Hence, our aims were to elucidate the changes of the carotid arterial structure and functions in patients with the HC. The purpose of the present study was to investigate the effect of pitavastatin calcium on artherosclerosis of carotid artery in patients with hypercholesteremia (HC).

Methods A total of 40 patients with $\mathrm{HC}$ were administered pitavastatin calcium $1 \mathrm{mg}$ or $2 \mathrm{mg}$ daily for 8 weeks and thirty healthy subjects were chosen as controls. Carotid atherosclerosis was evaluated by high-resolution B-mode ultrasonography $(5-\mathrm{MHz}$ linear array transducer; Sequia 512, Siemens). The right and left common carotid arteries (CCA) were assessed in the antero-oblique direction. For each study the following parameters were assessed and calculated using the following formulae, respectively: (1) IMT, (2): Vs, Vd, Vm, (3):Ep [Pressure-strain elastic modulus, $\mathrm{E} p=\{(\mathrm{SBP}-\mathrm{DBP}) /(\mathrm{Dd}-\mathrm{Ds}) \times$ $\mathrm{Dd}\}, \mathrm{Ep}^{*} \mathrm{Ep}^{*}=\mathrm{Ep} / \mathrm{DBP}(4) \beta:\{\beta=\mathrm{In}(\mathrm{SBP} / \mathrm{DBP}) /[(\mathrm{Dd}-\mathrm{Ds}) / \mathrm{Dd}]\},(5):$ AC $[$ Arterial compliance, $\mathrm{AC}=\pi(\mathrm{Ds} \times \mathrm{Ds}-\mathrm{Dd} \times \mathrm{Dd}) / 4 \times($ SBP-DBP $)]$, (6) RI:[RI=(Vs-Vd)/Vs], (7)PI:[PI=(Vs-Vd)/Vm], (8)plaque index.

RESULTS (1) As compared with healthy control group, IMT, $\beta$ in the HC group was significantly higher $(p<0.001)$, while Vs, RI, AC in the HC group was markedly lower $(p<0.01)$. (2) In HC patients, $\beta$ level was significantly reduced $(p<0.001)$, and, RI, AC were improved significantly ( $<<0.05, p<0.001$ respectively) 8 weeks after treatment as compared with those before treatment, however, no significant difference was noted in IMT.

Conclusions Our findings demonstrated that (1) Significant alterations to carotid arteries structure and functions were found in the HC patients, including thickened IMT, distended vessel diameter, reduced elasticity and augmented stiffness. (2) Carotid arterial functions were markedly ameliorated after treatment with pitavastatin calcium for 8 weeks.

\section{e0319 PREDICTING 30-DAY MORTALITY AMONG PATIENTS HOSPITALISED FOR DECOMPENSATED HEART FAILURE}

doi:10.1136/hrt.2010.208967.319

${ }^{1}$ Zhao Yusheng, ${ }^{1}$ Wu Xingli, ${ }^{1}$ Xue Qiao, ${ }^{1}$ Gao Lei, ${ }^{2}$ Lin Haili, ${ }^{1}$ Wang Shiwen. ${ }^{1}$ Institute of Geriatric Cardiology, Chinese Pla General Hospital; ${ }^{2}$ Chinese Pla General Hospital

Objective We investigated clinical correlates of in-hospital mortality and comorbidity of patients demonstrating heart failure progression in a large population.

Methods We included 6,949 patients with demonstrating heart failure who were hospitalised from the period of January 1, 1993, to December 31, 2007, at Chinese PLA General Hospital in Beijing. Hospital mortality and comorbidities were examined for the patients primarily admitted for decompensated HF.

Results The 30 -day in-hospital mortality was $5.4 \%$ in patients. Cox regression multivariate analysis showed that a history of cor pulmonale, stroke, renal failure, cirrhosis of liver-myocardial infarction, pneumonia, gastrointestinal bleeding and multiple organ dysfunction syndromes and age older than 65 years were the only independent predictors of in-hospital mortality. Using the regression coefficient as a benchmark, we calculated a convenient score. Nearly $23 \%$ of the patients with the score $>6$ died compared with only $1.2 \%$ of the patients with the score of 0 .

Conclusion Medical comorbidity at admission or age older than 65 years is an independent risk factor for 30-day mortality in patients with heart failure. The study illustrates that medical comorbidities at admission have proved to be a major prognostic marker for immediate poor outcome in the patients with heart failure. The score may help to identify patients who are more likely to have a risk of in-hospital mortality within 30-days.

\section{e0320 IMPACT OF PLAQUE MORPHOLOGY ON INTIMAL HYPERPLASIA AFTER STENTING ASSESSED BY OPTICAL COHERENCE TOMOGRAPHY}

doi:10.1136/hrt.2010.208967.320

Jin Qinhua, Chen Yundai, Akasaka, Mizukoshi, Kitabata, Liu Changfu, Tian Feng, Chen Lian, Sun Zhijun, Liu Hongbin, Wang Zhifeng, Guo Jun, Wang Jinda. Chinese Pla General Hospital

Objective The objective of this study was to evaluate whether the plaque morphology can affect the in-stent neointimal hyperplasia. 